\title{
Construction of a Chimeric Secretory IgA and Its Neutralization Activity against Avian Influenza Virus H5N1
}

\author{
Cun Li, ${ }^{1}$ Xiaoping An, ${ }^{1}$ Azeem Mehmood Butt, ${ }^{2}$ Baozhong Zhang, ${ }^{1}$ Zhiyi Zhang, \\ Xiaona Wang, ${ }^{1}$ Yong Huang, ${ }^{1}$ Wenhui Zhang, ${ }^{1}$ Bo Zhang, ${ }^{1}$ Zhiqiang Mi, ${ }^{1}$ and Yigang Tong ${ }^{1}$ \\ ${ }^{1}$ State Key Laboratory of Pathogen and Biosecurity, Beijing Institute of Microbiology and Epidemiology, Beijing 100071, China \\ ${ }^{2}$ Centre of Excellence in Molecular Biology (CEMB), University of the Punjab, Lahore-53700, Pakistan
}

Correspondence should be addressed to Zhiqiang Mi; zhiqiangmi.ime@gmail.com and Yigang Tong; tong62035@gmail.com

Received 7 September 2013; Accepted 7 January 2014; Published 13 February 2014

Academic Editor: Jiri Mestecky

Copyright (C) 2014 Cun Li et al. This is an open access article distributed under the Creative Commons Attribution License, which permits unrestricted use, distribution, and reproduction in any medium, provided the original work is properly cited.

Secretory immunoglobulin A (SIgA) acts as the first line of defense against respiratory pathogens. In this assay, the variable regions of heavy chain $(\mathrm{VH})$ and Light chain $(\mathrm{VL})$ genes from a mouse monoclonal antibody against H5N1 were cloned and fused with human IgA constant regions. The full-length chimeric light and heavy chains were inserted into a eukaryotic expressing vector and then transfected into $\mathrm{CHO} / \mathrm{dhfr}$-cells. The chimeric monomeric IgA antibody expression was confirmed by using ELISA, SDSPAGE, and Western blot. In order to obtain a dimeric secretory IgA, another two expressing plasmids, namely, pcDNA4/His A-IgJ and pcDNA4/His A-SC, were cotransfected into the $\mathrm{CHO} / \mathrm{dhfr}$-cells. The expression of dimeric SIgA was confirmed by using ELISA assay and native gel electrophoresis. In microneutralization assay on 96-well immunoplate, the chimeric SIgA showed neutralization activity against $\mathrm{H} 5 \mathrm{~N} 1$ virus on $\mathrm{MDCK}$ cells and the titer was determined to be $1: 64$. On preadministrating intranasally, the chimeric SIgA could prevent mice from lethal attack by using A/Vietnam/1194/04 H5N1 with a survival rate of $80 \%$. So we concluded that the constructed recombinant chimeric SIgA has a neutralization capability targeting avian influenza virus H5N1 infection in vitro and in vivo.

\section{Introduction}

Endemic highly pathogenic avian influenza virus (AIV) H5N1 in poultry has been present since the first occurrence in 1997 in Hong Kong. AIV H5N1 circulates in waterfowl and domesticated avian species and has evolved into multiple phylogenetically distinct genotypes and clades [1-3], with geographically distinct groups in each country. $\mathrm{H} 5 \mathrm{~N} 1$ viruses occasionally infect humans, with high case-fatality rates. These viruses have repeatedly crossed the species barrier and caused highly lethal human infections. The wide distribution of highly pathogenic AIV H5N1 is a global threat to human health [4-7]. Most deaths have occurred in young, previously healthy, adults or children. According to the latest WHO report [8], there have been 633 laboratory-confirmed highly pathogenic H5N1 AI cases worldwide from 2003 to 2013, with a mortality of $59.6 \%$.
For active immunization, vaccination would be ideal; however, there are some problems with avian influenza (AI) vaccines at present. There is no current pandemic of $\mathrm{AI}$ in humans, and therefore it is difficult to accurately assess the protective effects of any vaccine. Vaccines also have a major drawback because it would take several weeks to produce protective antibodies. This often reduces preventative effects and obstructs their effectiveness as emergency protection, especially in some high-risk groups. In contrast, passive immune agents can make up for the deficiencies of vaccines and can generate protective effects immediately after administration.

Research into passive immunity for AI prevention and treatment has been intensive in recent years. Animal experiments have shown that either polyclonal (serum, plasma) [911] or monoclonal [12-16] antibodies offer good protection against highly pathogenic AI. Meanwhile, many researchers 
TABle 1: Primers used in this assay.

\begin{tabular}{lr}
\hline Primer name & Primer sequence \\
\hline mVK-f-ATG & ATGGAGWCAGACACACTCCT \\
mVK-r & GGATACAGTTGGTGCAGCATC \\
mVH-f-ATG & ATGGRATGGAGCTGGATCTT \\
mVH-r & ATAGACAGATGGGGGTGTCGTTTTGGC \\
IGA-mVHhCHf & TCTCCTCAGCATCCCCGACCAGCCCCAA \\
IGA-mVHhCHr & TCGGGGATGCTGAGGAGACGGTGACTGA \\
IGA-Hf-EcoRI & CGGAATTCACCACCATGGGATGGAGCTGGATCT \\
IGA-Hr-Xbal & GCTCTAGATCAGTAGCAGGTGCCGTCCA \\
IGA-mVKhCKf & AATCAAACGAACTGTGGCTGCACCATCT \\
IGA-mVKhCKr & CCACAGTTCGTTTGATTTCCAGCTTGGT \\
IGA-Kf-EcoRI & CGGAATTCACCACCATGGAGACAGACACACTCCT \\
IGA-Kr-Xbal & GCTCTAGACTAACACTCTCCCCTGTTGAAGCTCTTTGTGA \\
\hline
\end{tabular}

have reported antibodies providing broad cross-protection against AIV H5N1 [12, 14, 17-19].

As a respiratory disease, AIV infection occurs via respiratory or digestive tract mucosa. Secretory IgA (SIgA), first identified in the 1960s, is a type of IgA antibody found in breast milk, gastrointestinal fluids, respiratory secretions, and genitourinary tracts. SIgA consists of two monomeric IgA units, which are associated with the J chain acquired during the process of polymerization in plasma cells just before secretion, along with the secretory component (SC) $[20,21]$. SIgA is considered the first-line defense in mucosal immunity and plays a critical role in preventing pathogen adhesion to host cells, thereby blocking dissemination and further infection. Because of its dimeric structure, SIgA has a higher functional affinity [22]. In vitro, SIgA is more resistant to proteases than serum IgA [23-25]. Its half-life is three times longer than IgG on mucosal surfaces, and it can provide a specific protective effect for at least 4 months [22]. The presence of the SC also gives SIgA special protective immunity activity. First, the SC has nonspecific activity against pathogenic microorganisms [26]. Second, via carbohydrate residues, SIgA can adhere to epithelial surfaces, forming a protective layer and effectively preventing invasion by a virus $[27,28]$. It would be of great significance to demonstrate the blocking effects of SIgA against AIV infection in the respiratory or digestive tracts.

Previous reports have shown that $\operatorname{IgA}$ can potentially be used for passive protection or therapeutic intervention on mucosal surfaces. IgA can act as a neutralizing antibody against pathogens and exotoxins, with better affinity than neutralizing antibodies of other classes [29]. Monoclonal IgA antibodies against respiratory syncytial virus were applied passively to the nasopharyngeal mucosa and prevented subsequent infection and pneumonia [30]. Passive oral delivery of IgA antibodies also protected against bacterial infections in the intestine of mice [31]. IgA has lower proteolytic stability without the bound SC $[23,24]$, and therefore it may be efficient to use purified SIgA as a passive treatment agent. In this study, we constructed a mouse and human derived SIgA and explored its feasibility in preventing $\mathrm{H} 5 \mathrm{~N} 1$ virus infection. Our results revealed that the recombinant SIgA could act as a preventative agent against $\mathrm{H} 5 \mathrm{~N} 1$ infection.

\section{Materials and Methods}

2.1. Reagents, Cells, and Virus. Restriction endonucleases and T4 Ligase for cloning were obtained from New England Biolabs (Beverly, MA, USA). Lipofectamine 2000 was purchased from Gibco BRL (Gaithersburg, MD, USA), with protein Aagarose, plasmid pcDNA4/His A, and zeocin from Invitrogen (Carlsbad, CA, USA). Dulbecco's Modified Eagle's Medium (DMEM) and Fetal Bovine Serum (FBS) were obtained from Hyclone (Logan, UT, USA). A hybridoma cell line secreting an anti-H5N1 (HA) antibody HA-9 was a gift from Dr. Chen (Beijing Institute of Microbiology and Epidemiology). A One-Step RT-PCR Kit was purchased from Takara (Dalian, China). The eukaryotic expression vector pEF-dhfr2a-NEO [32] was constructed in our laboratory. The $\mathrm{CHO}$ cells were cultured in DMEM containing 10\% fetal calf serum. MadinDarby canine kidney (MDCK) cells were purchased from the American Type Culture Collection (ATCC, Manassas, VA). Cells were used for a maximum of 25 passages and maintained in DMEM containing 5\% FBS, 2 mM L-glutamine, penicillin, and streptomycin (Gibco BRL). Cultures were incubated at $37^{\circ} \mathrm{C} / 5 \% \mathrm{CO}_{2}$ in a humidified atmosphere. Aliquots of H5N1 influenza A virus A/Vietnam/1194/04 grown in embryonated eggs was stored at $-70^{\circ} \mathrm{C}$. The $50 \%$ tissue culture infectious dose $\left(\mathrm{TCID}_{50}\right)$ was determined by titration in MDCK cells.

2.2. Cloning of the HA-9 Variable Region and Construction of the Vector Expressing Heavy Chain. Total RNA was extracted from hybridoma cells using Trizol (Invitrogen), and the variable region gene was cloned by using a Takara One-Step RT-PCR Kit according to the manufacturer's instructions. The variable region of the HA-9 light chain (mVL) gene was cloned with primers mVK-f-ATG and mVK-r. The heavy chain variable gene $(\mathrm{mVH})$ was cloned using primers $\mathrm{mVH}-\mathrm{f}-$ ATG, and mVH-r. PCR products were recovered and inserted into pMD18-T, then verified by sequencing. Sequences of the primers used in this assay were shown in Table 1. 
Plasmid pGEM-T-Easy-IGHA [33] was digested with EcoRI. The $1400 \mathrm{bp}$ fragment carrying the IgA2 heavy chain constant region gene (IGHA2) was recovered. $\mathrm{mVH}$ was recovered from pMD18-T-mVH by digesting with EcoRI and SalI. A mixture of IGHA2 and $\mathrm{mVH}$ was amplified by overlap PCR with primers IGA-mVHhCHf, IGA-mVHhCHr, IGAHf-EcoRI, and IGA-Hr-XbaI. Products of the primary PCR were used as the template for amplifying the full-length chimeric IgA in the secondary PCR with primers IGA-HfEcoRI and IGA-Hr-XbaI. The PCR products were recovered and then cloned into pMD18-T. The resulting recombinant plasmid was designated pT-CHI-mVH-IGHA, then verified by sequence analysis. A fragment $(1.80 \mathrm{~kb})$ was recovered by digesting pT-CHI-mVH-IGHA with EcoRI and XbaI. This fragment was then ligated into EcoRI/XbaI-treated pEFdhfr2a-NEO, creating the expression vector pEF-dhfr2aNEO-IGA-chi-H.

2.3. Construction of the Vector Expressing Light Chain. pGEM-T-Easy-CK was digested with EcoRI, and a $400 \mathrm{bp}$ fragment encoding the light chain constant region (CK) was recovered. $\mathrm{mVK}$ was recovered from $\mathrm{pMD} 18-\mathrm{T}-\mathrm{mVK}$ by digesting with EcoRI and SalI. The mixture of CK and $\mathrm{mVK}$ was amplified by overlap PCR with primers IGA-mVKhCKf, IGA-mVKhCKr, IGA-Kf-EcoRI, and IGA-Kr-XbaI. Products of the primary PCR were used as the template to amplify the full-length light chain of chimeric IgA in the secondary PCR (IGA-Kf-EcoRI and IGA-Kr-XbaI). PCR products were recovered and cloned into pMD18-T to yield pT-CHI-mVKIGK, which was verified by sequence analysis. A $700 \mathrm{bp}$ fragment was recovered by digesting pT-CHI-mVK-IGK with EcoRI and XbaI. The fragment comprising the light chain DNA was then ligated into EcoRI/XbaI-treated pEFdhfr2a-NEO, creating expression vector pEF-dhfr2a-NEOIGA-Kappa.

\subsection{Construction of the Vectors Expressing SC or J Chain.} According to previously published techniques [33, 34], we obtained the full-length pIgR gene and pcDNA4/His A-pIgR [33]. The human SC is composed of the first 585 amino acid residues of the polymeric Ig receptor. Using a published protocol, the "DREAM" technique [35], we mutated the intracellular region of $\mathrm{pIgR}$, thereby generating $\mathrm{pcDNA} 4 / \mathrm{His}$ A-SC expression vector. Using the same technique [33], we acquired the IgJ gene, ligated it into the expression vector $\mathrm{pcDNA} 4 / \mathrm{His} \mathrm{A}$, and produced the expression plasmid, pcDNA4/His A-IgJ. All the above recombinant vectors were illustrated in diagram (Figure 1).

2.5. Establishment of IgA- and SIgA-Expressing CHO Cell Line. The CHO dhfr-cells were cultured in DMEM supplemented with $10 \% \mathrm{FBS}, 100 \mu \mathrm{M}$ hypoxanthine, and $16 \mu \mathrm{M}$ thymidine. The cell concentration was adjusted to $2 \times 10^{5}$ cells $/ \mathrm{mL}$ by diluting with DMEM containing 10\% FBS and seeded into six-well plates. Lipofectamine $2000(4 \mu \mathrm{L})$ was mixed with pEF-dhfr2a-NEO-IGA-chi-H $(2 \mu \mathrm{g})$ and pEF-dhfr2a-NEOIGA-Kappa $(2 \mu \mathrm{g})$, respectively, in a final volume of $200 \mu \mathrm{L}$ of DMEM. The mixture was incubated for a further $20 \mathrm{~min}$ then gently added to one well of the six-well plate. After a $6 \mathrm{~h}$ incubation at $37^{\circ} \mathrm{C} / 5 \% \mathrm{CO}_{2}$, cells were fed with DMEM lacking hypoxanthine and thymidine, but containing $10 \%$ dialyzed FBS. Cells stably secreting IgA were screened in 96well culture dishes, with selection continuing for 3 weeks. Clones expressing IgA were screened by using ELISA. A cell clone, which produced the most IgA, was amplified to serve as the recipient cell for subsequent transfections with pcDNA4/His A-SC and pcDNA4/His A-IgJ. Selection was carried out in the presence of $500 \mathrm{mg} / \mathrm{mL}$ Zeocin over 3 weeks. Cells producing SIgA were then amplified to be selected in methotrexate (MTX), the concentration of which was gradually increased.

2.6. Detection of Produced IgA and Determination of Its Antigen Binding Capacity. Sandwich ELISA was conducted to detect the production of IgA. A mouse anti-human IgA $\alpha$ chain-specific monoclonal antibody (Sigma-Aldrich, diluted $1: 3000$ in $0.05 \mathrm{M} \mathrm{Na}_{2} \mathrm{CO}_{3}$ buffer, $\mathrm{pH}$ 9.6) was used to coat the wells $(50 \mu \mathrm{L} /$ well $)$ of Costar immunoplates overnight at $4^{\circ} \mathrm{C}$. The plates were blocked with $5 \%$ skim milk in PBS followed by washing three times with PBS containing 0.05\% Tween 20 (PBST). IgA supernatant $(50 \mu \mathrm{L})$ and human IgA (saliva) were then added to the wells and incubated for $2 \mathrm{~h}$ at $37^{\circ} \mathrm{C}$. After extensive washing with PBST, HRP-conjugated goat anti-human IgA (InvivoGen) diluted 1:2500 in 2\% skim milk in PBST was added $(50 \mu \mathrm{L} /$ well). After incubation at $37^{\circ} \mathrm{C}$ for $1 \mathrm{~h}$, further washes were followed by the addition of $3,3^{\prime}, 5,5^{\prime}$-tetramethylbenzidine for $15 \mathrm{~min}$ at $37^{\circ} \mathrm{C}$. The assay was stopped with the addition of $50 \mu \mathrm{L}$ of $2 \mathrm{M} \mathrm{H}_{2} \mathrm{SO}_{4}$ and the absorbance at $450 \mathrm{~nm}$ measured immediately. The antigen binding capacity of the antibodies was determined by using a sandwich ELISA as described above. The capture antigen for this ELISA was A/Vietnam/1194/04 H5N1 HA antigen ( $500 \mathrm{ng} /$ well), purified from the lysate of virus. The Bovine Serum Albumin (BSA) was coated on the wells as negative control.

2.7. SIgA Western Blot Analysis. CHO culture supernatant $(1 \mathrm{~mL})$ was incubated with rabbit anti-human $\alpha$-chainspecific antibody ( $30 \mu \mathrm{L}$, Sigma-Aldrich) overnight at $4^{\circ} \mathrm{C}$. Protein-A sepharose ( $50 \mu \mathrm{L}$, Sigma-Aldrich) equilibrated in PBS was added and the mixture incubated for $4 \mathrm{~h}$ at $4^{\circ} \mathrm{C}$ then centrifuged $\left(3000 \times \mathrm{g}, 4^{\circ} \mathrm{C}, 1 \mathrm{~min}\right)$. The resulting pellet was washed three times with $1 \mathrm{~mL}$ of PBS. The immunoprecipitated protein was separated by reduced or nonreduced SDS-PAGE, and then transferred to polyvinylidene difluoride membranes. Membranes were blocked overnight at $4^{\circ} \mathrm{C}$ by incubating with $10 \%$ skim milk in PBST, then incubated for $2 \mathrm{~h}$ at $37^{\circ} \mathrm{C}$ with the following primary antibodies, respectively: mouse anti-human IgA, $\alpha$-chain specific $(1: 3000$; Abcam, Cambridge, MA, USA); mouse anti-human, $\kappa$-chain specific (1:3000; Sigma Aldrich); mouse anti-human, J chain specific (1:2500; Abcam); and mouse anti-human, SC specific (1:3000; Sigma Aldrich). Bound antibodies were detected with an HRP-conjugated goat anti-mouse IgG ( $1: 3000$; Sigma Aldrich) in combination with enhanced chemiluminescent reagents (Millipore, Bedford, MA, USA). 


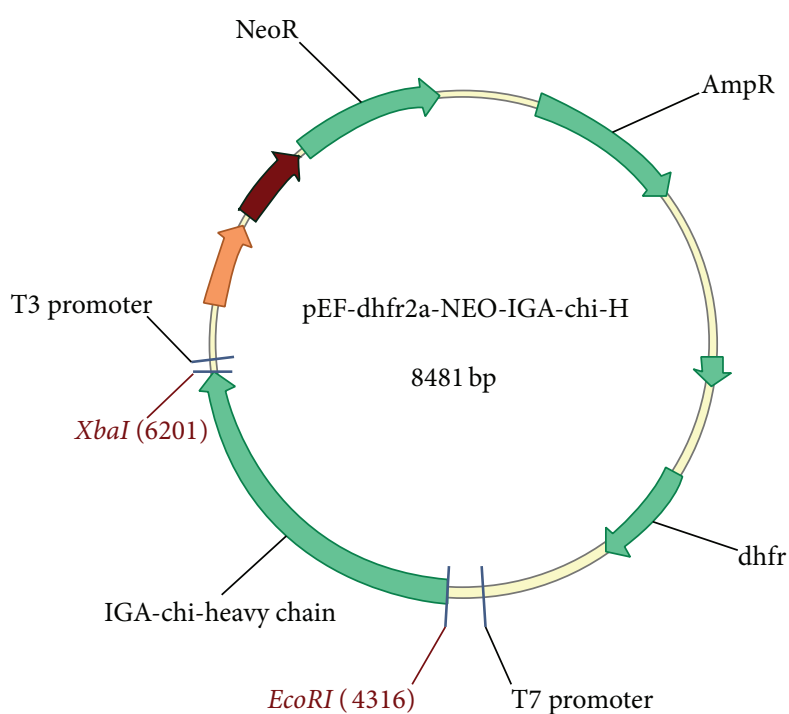

(a)

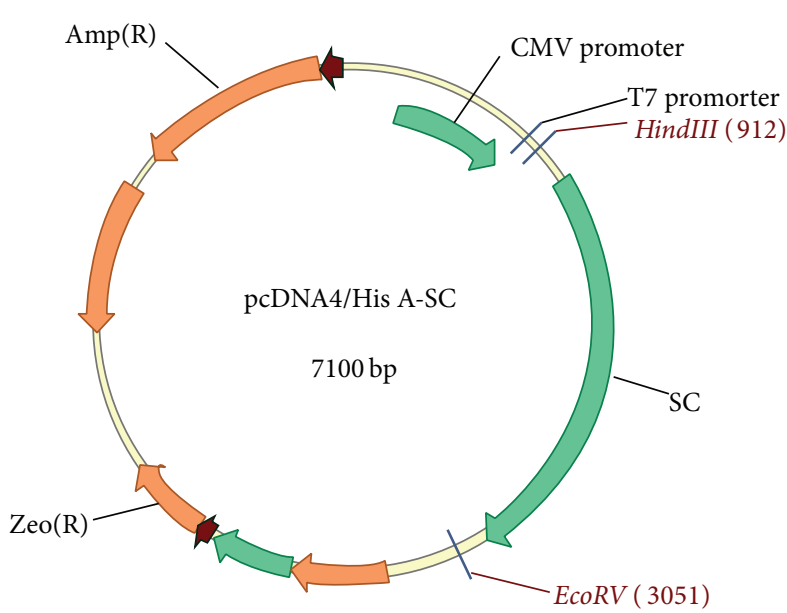

(c)

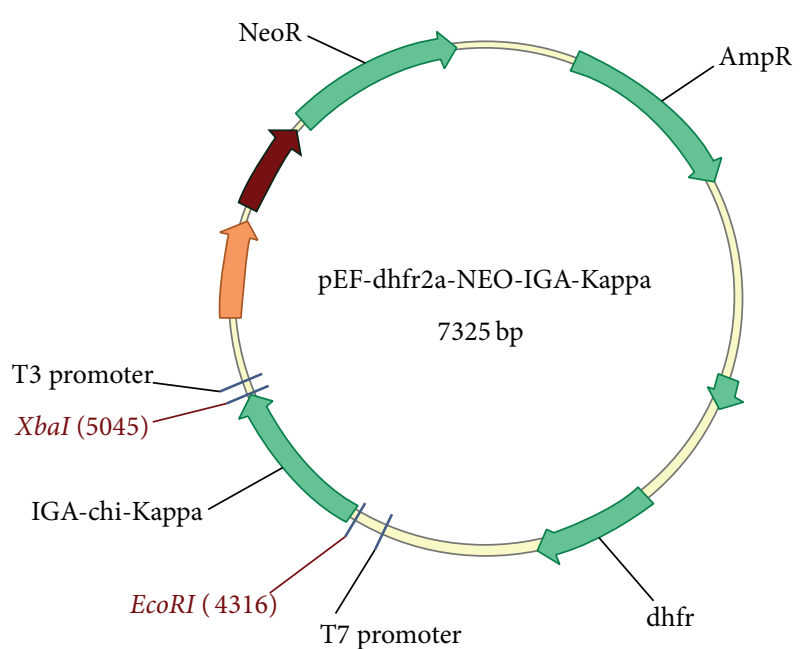

(b)

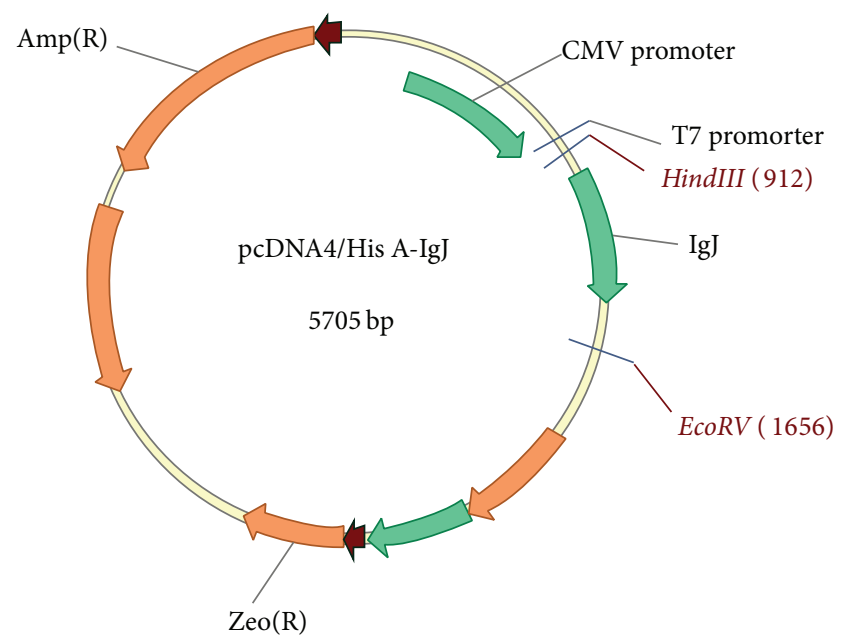

(d)

FIGURE 1: Schematic of the expression vectors. pEF-dhfr2a-NEO-IGA-chi-H and pEF-dhfr2a-NEO-IGA-Kappa contain the marker neoR and dhfr-. pcDNA4/His A-SC and pcDNA4/His A-IgJ contain the ORF for zeocin resistance (zeoR). The restriction sites used for the construction of the vectors are indicated.

2.8. SIgA Fermentation and Purification. Monoclonal SIgA cells were adapted to serum-free medium CD CHO medium (Gibco, Carlsbad, CA, USA) with $125 \mathrm{nM}$ MTX over 2 months. The concentration of DMEM and dialysis serum was gradually reduced until cells were fully adapted to $\mathrm{CD} \mathrm{CHO}$ medium. The adapted cell lines were grown in suspension cultures for 2-4 weeks in $\mathrm{CD}$ CHO medium containing $125 \mathrm{nM}$ MTX. Culture supernatant was collected and purified using Pierce Protein L Chromatography Cartridges (Thermo Scientific, Hudson, NH, USA). After centrifugation $(5000 \times \mathrm{g}$, $10 \mathrm{~min}$ ), the supernatant was passed through a $0.45 \mu \mathrm{m}$ filter. The filtrate was then loaded onto a Protein L affinity column and the bound antibody was eluted with buffer ( $0.2 \mathrm{M} \mathrm{Na}_{2} \mathrm{HPO}_{4}, 0.1 \mathrm{M}$ citric acid $\mathrm{pH}$ 3.0). The antibodies were concentrated using a Centriplus YM-100 ultrafiltration tube (Pierce Chromatography Cartridges Protein-L) and the buffer replaced with sterile PBS. The resulting solution was aliquoted and stored at $-20^{\circ} \mathrm{C}$ until required.

2.9. Neutralization Assay and Statistical Analysis. All microneutralization assays were performed on MDCK cells, with cells used for a maximum of 25 passages. The virus $\mathrm{TCID}_{50}$ was also determined by titration on these cells. Briefly, serial 10 -fold dilutions of virus were made in DMEM containing $1 \%$ BSA, and a $100 \mu \mathrm{L}$ of each dilution was dispensed into 96-well plate. Freshly trypsinized MDCK cells were adjusted to a concentration of $1.5 \times 10^{5}$ cells $/ \mathrm{mL}$ and aliquots $(100 \mu \mathrm{L})$ added to each well. Each dilution was assayed in triplicate. Plates were covered and incubated at $37^{\circ} \mathrm{C} / 5 \% \mathrm{CO}_{2}$. Wells where a cytopathic effect (CPE) was observed were considered to be positive for virus growth. 
The $\mathrm{TCID}_{50}$ was calculated by the method of Reed and Muench [36].

Purified recombinant SIgA $(10 \mathrm{mg} / \mathrm{mL})$ was serially diluted 2-fold, with $50 \mu \mathrm{L}$ of each dilution added, in triplicate, to wells of a 96-well plate. Virus $(100 \mu \mathrm{L})$ containing 100 $\mathrm{TCID}_{50}$ was added to each well. Freshly trypsinized MDCK cells were adjusted to a concentration of $1.5 \times 10^{5}$ cells $/ \mathrm{mL}$ and aliquots $(100 \mu \mathrm{L})$ added to each well. The reciprocal of the last dilution at which infection was completely blocked was determined as the microneutralization titer of the virus stock.

All animal studies were approved by the Institutional Animal Care and Use Committee at Beijing Institute of Microbiology and Epidemiology, Beijing, China, and performed according to institutional guidelines for animal welfare. Mice were housed with 8 per cage and maintained on a 12 hour light/ dark cycle (lights on at 7:00 am) with continuous access to food and water. At the end of animal study mice were sacrificed by $\mathrm{CO}_{2}$ asphyxiation in accordance with the guidelines. Female BALB/c mice ( 6 weeks old) were kept in biosafety level 3 housing. All experimental protocols followed the standard operating procedures of the biosafety level 3 animal facilities. Aliquots of influenza A H5N1 A/Vietnam/1194/04 stocks were grown in embryonated eggs. Virus-containing allantoic fluid was harvested and stored in aliquots at $-70^{\circ} \mathrm{C}$. The $\mathrm{LD}_{50}$ was determined in mice following serial dilution of the virus stock. For the viral challenge, $10 \mathrm{LD}_{50}$ were used in all experiments. Infection was established by intranasal inoculation of mice anesthetized with ketamine. The mice of negative control group (SIgA) were administrated with $20 \mu \mathrm{L}$ of saline and $20 \mu \mathrm{L}$ of SIgA $(10 \mathrm{mg} / \mathrm{mL})$, and those of positive control group (Challenge) were given $20 \mu \mathrm{L}$ of saline and $20 \mu \mathrm{L}$ of virus $\left(10 \mathrm{LD}_{50}\right)$. Mice in Group 1 (SIgA + Challenge) were first administrated with $20 \mu \mathrm{L}$ of SIgA, and then $20 \mu \mathrm{L}$ of virus $\left(10 \mathrm{LD}_{50}\right) 2 \mathrm{~h}$ later. Mice in group 2 (Challenge $\left.+\operatorname{SIgA}\right)$ were first administrated with $20 \mu \mathrm{L}$ of virus $\left(10 \mathrm{LD}_{50}\right)$, and then $20 \mu \mathrm{L}$ of SIgA $2 \mathrm{~h}$ later. Each group contains 8 mice.

Statistical analysis of the SIgA binding capacity data was performed by using an independent $t$-test. Survival data was analyzed by using a Kaplan-Meier survival analysis with a log-rank method of statistics. $P<0.05$ was considered significant.

\section{Results}

3.1. Construction of SIgA Expression Vectors. The variable regions of the heavy $(\mathrm{VH})$ and light $(\mathrm{VL})$ chain genes of the anti-H5N1 HA neutralizing monoclonal antibody were cloned by RT-PCR [37]. These genes were analyzed by using the England Bioinformatics Institute website (IMGT/VQUEST). The signal peptides for VH and VL were predicted by using artificial neural networks and hidden Markov models on the Danish Centre for Biological Sequence Analysis website (http://www.cbs.dtu.dk/services/SignalP/). The results indicated that $\mathrm{VH}$ and $\mathrm{VL}$ were derived from mouse immunoglobulin genes. The VH comprised $414 \mathrm{bp}$, with the first 57 nucleotides encoding a signal sequence. The genes for $\mathrm{VH}, \mathrm{D}$, and $\mathrm{J}$ were originally derived from mouse antibody

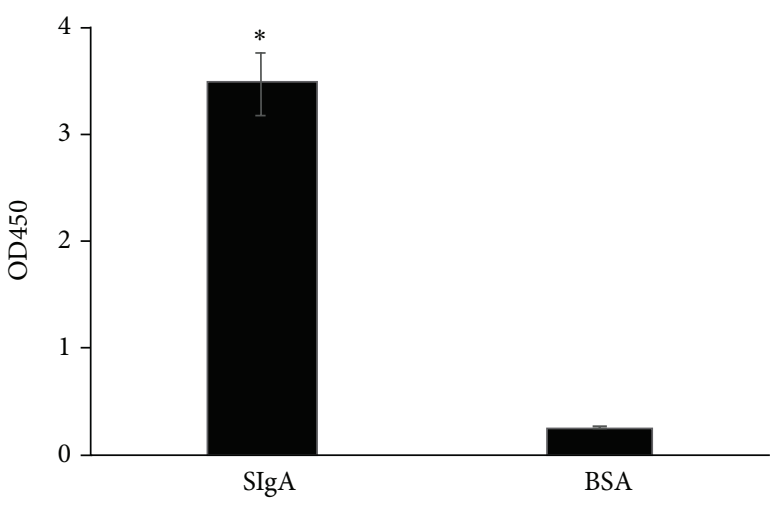

FIGURE 2: ELISA analysis of H5N1 HA antigen binding capacity of SIgA. H5N1 HA or BSA was coated on 96-well plate as the capture antigens. The produced SIgA was added as the primary antibody, and the secondary antibody was HRP-conjugated goat anti-human $\operatorname{IgA}$. Data were the average of three independent experiments and shown as mean \pm SD. Significance was determined by using independent $t$-test $\left({ }^{*} P<0.05\right)$.

genes IgHV I, IgHV IV, and IgHV II, respectively. The VL contains $393 \mathrm{bp}$, with the first 60 nucleotides encoding a signal sequence. The $\mathrm{V}$ region and J genes were derived from mouse antibody genes IgKV III and IgKJ I, respectively. To improve production of IgA in eukaryotic cells, the cloned IGHA sequence contained all introns [38].

3.2. Screening and Antigen Binding of SIgA. Sandwich ELISA was used to screen the cell clones expressing IgA and SIgA (data not shown), and the clone 6 which produces the most amount of SIgA was selected in the following assays. The capacity of the produced SIgA binding to antigens was evaluated by using the Sandwich ELISA, and the H5N1 HA was used as a capturing antigen. The results showed that the produced recombinant SIgA could bind to the H5N1 HA antigen (Figure 2).

3.3. SIgA-Specific Western Blot Analysis. Recombinant chimeric SIgA produced by cells was detected by westernblotting under reduced and non-reduced conditions. The Recombinant chimeric SIgA was shown to consist of four subunits: an IgA $\alpha$ chain $(55 \mathrm{kDa})$; a kappa light chain $(25 \mathrm{kDa})$; a J chain $(17 \mathrm{kDa})$; and the SC $(66 \mathrm{kDa})$. These four subunits were similar to those observed from human saliva SIgA (Figure 3). Results of SDS-PAGE under nonreducing conditions demonstrated that the recombinant SIgA presented as different composed patterns and was exactly the same as naturally-secreting SIgA (Figure 4). The band of H4L4JSC is the complete SIgA molecule. The band of H4JSC/H4L4J is composed of four IgA $\alpha$-chains, J chain and secretory component or of four IgA $\alpha$-chains, four $\kappa$-chains, and J chain. The band of HJSC/H2L is composed of one IgA $\alpha$-chain, J chain, and secretory component or of two IgA $\alpha$-chains and one $\kappa$-chain. The band of HLJ is composed of one IgA $\alpha$-chain, one $\kappa$-chain and J chain. The band of LJ is 


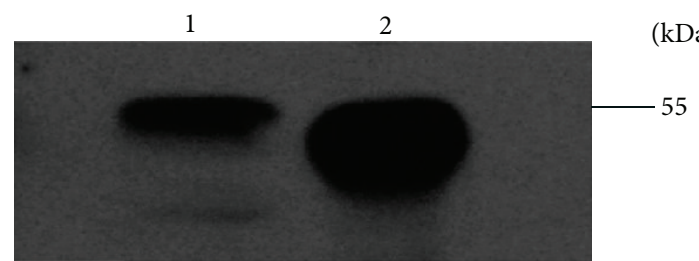

(a)

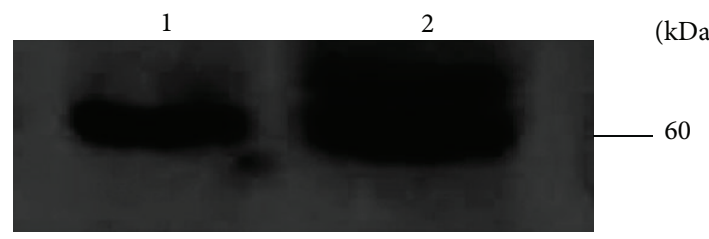

(c)

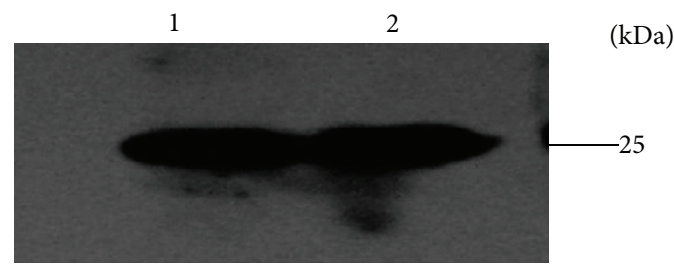

(b)

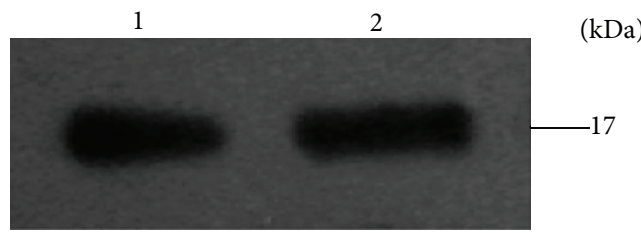

(d)

FIGURE 3: Western blot analysis of recombinant SIgA under reduced conditions. Transferred proteins were incubated with various specific primary antibodies. (a) Mouse anti-human IgA $\alpha$-chain. (b) Mouse anti-human $\kappa$-chain. (c) Mouse anti-human SC. (d) Mouse anti-human J chain. HRP-conjugated goat anti-mouse IgG was used as secondary antibody. Lane 1: human saliva (positive control); 2: produced SIgA.

composed of one $\kappa$-chain, and J chain. The band of H or SC means the monomer of $\operatorname{IgA} \alpha$-chain or secretory component.

3.4. Purification of SIgA. Recombinant SIgA was purified from cell culture supernatants, yielding approximately $25 \mathrm{mg}$ of antibody per liter of supernatant. The final preparation was diluted in sterile PBS to $10 \mathrm{mg} / \mathrm{mL}$. Using SDS-PAGE under non-reducing conditions; analysis of the purified antibody preparation demonstrated that the most abundant protein band was the purified SIgA, and the expected molecular size of the complex was $400 \mathrm{kDa}$ (Figure 5). Other abundant protein bands were present at $200 \mathrm{kDa}$, which might be corresponding to monomers of IgA, or degraded products of the antibody preparation.

3.5. Neutralization Assay. The obvious CPE was observed around $48 \mathrm{~h}$ post-infection (p.i.). At $96 \mathrm{~h}$ p.i., CPE was evaluated and calculated for all wells. The $\mathrm{TCID}_{50}$ of the virus stock in MDCK cells was $10^{-6.5}$. When challenged with $100 \mathrm{TCID}_{50}$ of virus, administration of $50 \mu \mathrm{L}$ of a 64 fold dilution of purified SIgA conferred complete protection at $96 \mathrm{~h}$ p.i. Therefore, the microneutralization titer of the SIgA solution was designated as 64 . To examine the protective efficacy of the produced recombinant SIgA in vivo, we challenge the mice with a lethal dose of influenza A H5N1 A/Vietnam/1194/04 then administrated the mice with SIgA before or after challenging. When the mice in positive control group were infected with a dose of $10 \mathrm{LD}_{50}$ of virus intranasally, they all died within 6 days p.i. In the negative control group, when administrated with SIgA alone, all the mice survived until sacrificed at 14th day. In SIgA + Challenge group, preadministration with SIgA provided a protection with survival rate of $80 \%$. However, all mice in the group of Challenge + SIgA, in which the mice were administrated with SIgA after challenging, died at 7th day after infection. KaplanMeier analysis showed that pretreatment with SIgA could effectively prevent mice from lethal challenging (Figure 6) $(P<0.001)$.

\section{Discussion}

Antiviral drugs or vaccines are usually used to treat Influenza Virus infection. However, due to the prone mutation of Influenza Virus's genome, the virus easily acquires resistance to those available drugs. Using a vaccine to prevent AI would take 1-2 weeks to produce protective antibodies, during which time the virus could spread rapidly and cause severe health problems. So, developing a suitable agent for emergent prevention is prerequisite to control AI. The major invading routes of Avian Influenza Virus are the respiratory or digestive tract mucosa. Therefore, an agent targeting AIV on mucosa might bring effective protection in restricting AIV spreading, especially before the earliest stages of replication. In this study, we developed a chimeric SIgA as a passive agent to prevent the Avian Influenza H5N1 for the first time. A cell lines stably producing recombinant chimeric SIgA targeting AIV was constructed and the antiviral activity of the produced SIgA was evaluated in vitro and in vivo.

Production of SIgA normally requires the cooperation of two different cell types in the body. The heavy and light chains produced in plasma cells were assembled into IgA, which is on association with polymeric immunoglobulin receptor (pIgR) during transcytosing across the basolateral epithelial cells lining on the mucosa. The cytoplasmic and transmembrane region of the receptor was proteolytically cleaved, the truncated receptor termed secretory component (SC) and released from the receptor together with dimeric IgA. Previous experiments have shown that it is possible to assemble a functional SIgA molecule in vitro [22, 37, 39-41]. To clone the genes encoding SIgA subunits, highly specific and efficient primers (Table 1) were designed to amplify the exons directly from extracted genomic DNA. Sequence analysis confirmed that the cloned sequences were 


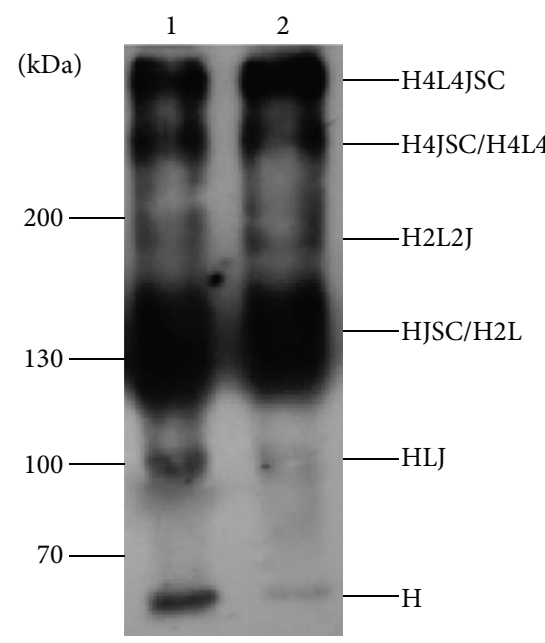

(a)

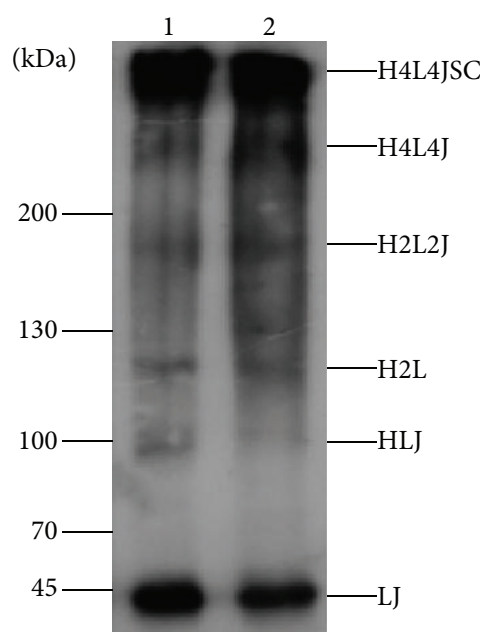

(c)

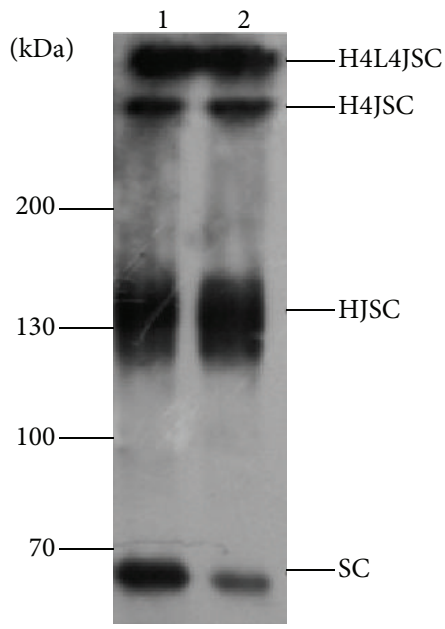

(b)

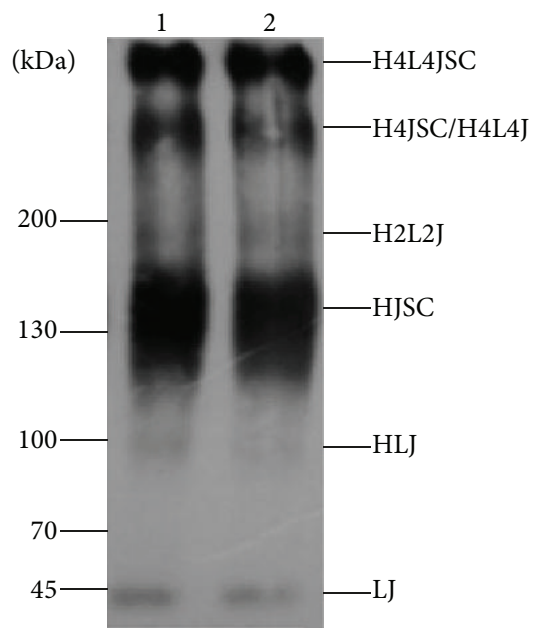

(d)

FIGURE 4: Western blot analysis of recombinant SIgA under nonreducing conditions. Transferred proteins were incubated with different primary antibodies to confirm the various polymeric forms of recombinant SIgA. (a) Mouse anti-human IgA $\alpha$-chain antibody. (b) Mouse anti-human SC antibody. (c) Mouse anti-human $\kappa$-chain antibody. (d) Mouse anti-human J chain antibody. HRP-conjugated goat antimouse IgG was used as secondary antibody. Lane 1: produced SIgA; 2: human saliva (positive control). H4L4JSC (complete SIgA molecule), H4JSC/H4L4J (four IgA $\alpha$-chains, J chain, and secretory component or four IgA $\alpha$-chains, four $\kappa$-chains, and J chain), HJSC/H2L (one IgA $\alpha$-chain, J chain, and secretory component or two IgA $\alpha$-chains and one $\kappa$-chain), HLJ (one IgA $\alpha$-chain, one $\kappa$-chain, and J chain), LJ (one $\kappa$-chain and J chain), and $\mathrm{H}$ or SC (monomer of IgA $\alpha$-chain or secretory component).

identical to the relative entries in the GenBank database. The "Genomic DNA Splicing" technique avoids RNA preparation and reverse transcription steps, and the entire assembly process can be finished within hours [33]. Because genomic DNA is more stable than RNA, it would be a more practical cloning strategy for many genes, especially for those that are very large where it is difficult to generate full-length cDNAs.

Heterologous protein expression is a complex interactive process between the vector and host cells. It is imperative to build a suitable vector to achieve the highest possible levels of protein expression. The eukaryotic expression vector used in this study, pEF-dhfr2a-NEO, was developed in our laboratory. This vector has many characteristics suitable for high-level expression of cloned DNA, including strong promoters, an SV40 late polyadenylation signal, introns, and two selectable markers. The vector has both CMV and human elongation factor 1 (EF1)- $\alpha$ promoter regions. The human CMV promoter is universally recognized as a strong promoter [38]. The EF1- $\alpha$ promoter (pEF) was cloned from the chromosome of human fibroblast cells [42]. EF1 is indispensable in protein synthesis and has a relatively high intracellular concentration. One study has shown that $\mathrm{pEF}$ is one of the strongest promoters discovered [43]. Polyadenylation has been shown to enhance RNA stability and translation [44]. Our vector contains an efficient SV40 late poly (A) signal downstream of the target gene [44-46], ensuring expression of foreign genes. Artificial chimeric introns can be used to reduce the chance of splicing and facilitate gene expression of 




FIGURE 5: SDS-PAGE analysis of purified SIgA under non-reducing conditions. Lane 1: protein molecular weight marker; 2: purified SIgA antibody.

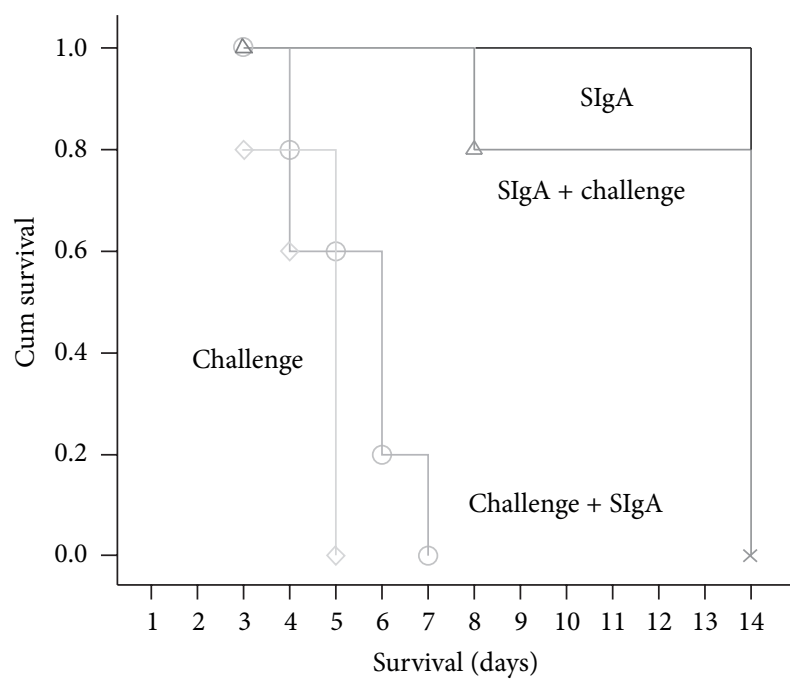

FIGURE 6: Survival analysis of BALB/c mice with different treatment. Kaplan-Meier survival analysis was used to determine the survival probability among the different groups. Mean survival days of the mice in Group 1 (SIgA + Challenge) was $12 \pm 1$ days versus $5 \pm 1$ days in Group 2 of Challenge + SIgA $(P<0.0001) \cdot n=8$.

cDNAs to a certain extent [47-49]. The vector pEF-dhfr2aNEO includes an artificial intron in upstream of the target gene to avoid incorrect splicing and promote high levels of constitutive expression of the cloned DNA in mammalian cells. There are two selectable markers in pEF-dhfr2a-NEO, one of which is the dihydrofolate reductase (DHFR) gene [50]. The DHFR amplification system has become very popular for use in developing recombinant $\mathrm{CHO}$ cell lines. The DHFR system allows the gene of interest to be amplified many times by gradually increasing the concentration of MTX, an inhibitor of the DHFR enzyme. Utilizing this system can lead to increased levels of recombinant protein expression [51].

According to the patterns corresponding to H4L4JSC, H2L2, and HL complexes, the SIgA ratio in cell culture supernatants was much higher than that observed for $\operatorname{dIgA}$ and mIgA (Figure 4). The presence of bands that reacted with anti- $\alpha$, anti- $\kappa$, anti-J, and anti-SC antibodies in high molecular weight species (H4L4J and H4L4JSC) confirms the covalent association of the light and J chains and the SC with heavy chains (Figure 4), which has been demonstrated previously $[22,37,41]$. These results indicated that we had successfully expressed the four polypeptides which efficiently assembled into entire SIgA antibody molecules in engineered $\mathrm{CHO}$ cells. The antigen-binding capacity of recombinant SIgA was measured by ELISA, and we demonstrated that SIgA had a strong capacity to bind antigens.

Recombinant SIgA was purified and the microneutralization assays were conducted by using the purified SIgA. When challenged with $100 \mathrm{TCID}_{50}$ of virus, administration with $50 \mu \mathrm{L}$ of a 64 -fold dilution of SIgA conferred complete protection at $96 \mathrm{~h}$ p.i. The microneutralization assay showed that SIgA had neutralization activity in vitro. On preadministrating with the purified SIgA, it can prevent $80 \%$ of the mice from lethal challenging; however, administration after infection could not bring effective protection to mice. It is not difficult to understand that preadministration would block virus entering the epithelial cells on mucosa. In contrast, when the virus finished the entry process, the SIgA locating on the surface of mucosa would not function to neutralize it. So we suggest that early administration is pivotal for SIgA functioning. Whether human components from the chimeric SIgA have effects on its potential efficacy was another important concern in designing chimeric antibody. The recombinant SC fragment used in this paper was of human origin, which had been proved to retain the function of directing SIgA distribution in the mucosa of mice [27]. Although the chimeric IgA exhibited the ability of binding to $\mathrm{HA}$ from Influenza virus $\mathrm{H} 5 \mathrm{~N} 1$ and neutralizing the virus in vitro, generally the chimeric antibody cannot retain one hundred percentage activity compared with its parental IgG molecule and this might influence its protective efficacy in mice to some extent. In conclusion, we have developed a recombinant anti-H5N1 SIgA in engineered CHO cells in this assay, which had a neutralizing capacity against influenza A H5N1 A/Vietnam/1194/04 in vitro and a protective capacity when administrated before challenging in vivo. Therefore, the developed SIgA in this research deserved to be further explored for application in emergent immunization.

\section{Conflict of Interests}

The authors declare that there is no conflict of interests regarding the publication of this paper.

\section{Authors' Contribution}

Cun Li and Xiaoping An contributed equally to this work. 


\section{Acknowledgments}

This paper was supported by Grants from the National Natural Science Foundation of China (no. 81072350 and 81072250), the China Mega-Project on Major Drug Development (no. 2011ZX09401-023), the China Mega-Project on Infectious Disease Prevention (no. 2011ZX10004-001), and the State Key Laboratory of Pathogen and BioSecurity Program (no. SKLPBS1113).

\section{References}

[1] Y. Guan, J. S. M. Peiris, A. S. Lipatov et al., "Emergence of multiple genotypes of $\mathrm{H} 5 \mathrm{~N} 1$ avian influenza viruses in Hong Kong SAR," Proceedings of the National Academy of Sciences of the United States of America, vol. 99, no. 13, pp. 8950-8955, 2002.

[2] D. J. Smith, A. S. Lapedes, J. C. De Jong et al., "Mapping the antigenic and genetic evolution of influenza virus," Science, vol. 305, no. 5682, pp. 371-376, 2004.

[3] G. J. Smith, X. H. Fan, J. Wang et al., "Emergence and predominance of an $\mathrm{H} 5 \mathrm{~N} 1$ influenza variant in China," Proceedings of the National Academy of Sciences of the United States of America, vol. 103, no. 45, pp. 16936-16941, 2006.

[4] K. Stöhr and M. Esveld, "Will vaccines be available for the next influenza pandemic?" Science, vol. 306, no. 5705, pp. 2195-2196, 2004.

[5] J. G. Bartlett and F. C. Hayden, "Influenza A (H5N1): will it be the next pandemic influenza? Are we ready?" Annals of Internal Medicine, vol. 143, no. 6, pp. 460-462, 2005.

[6] A.-N. Abdel-Ghafar, T. Chotpitayasunondh, Z. Gao et al., "Update on avian influenza A (H5N1) virus infection in humans," The New England Journal of Medicine, vol. 358, no. 3 , pp. 220-273, 2008.

[7] T. M. Uyeki, "Human infection with highly pathogenic avian influenza A (H5N1) virus: review of clinical issues," Clinical Infectious Diseases, vol. 49, no. 2, pp. 279-290, 2009.

[8] WHO, 2013, http://www.who.int/influenza/human_animal_ interface/ EN_GIP_20130705CumulativeNumberH5N1cases_2 .pdf.

[9] M. I. Shahzad, K. Naeem, M. Mukhtar, and A. Khanum, "Passive immunization against highly pathogenic Avian influenza virus (AIV) strain $\mathrm{H} 7 \mathrm{~N} 3$ with antiserum generated from viral polypeptides protect poultry birds from lethal viral infection," Virology Journal, vol. 5, article 144, 2008.

[10] B. Zhou, N. Zhong, and Y. Guan, "Treatment with convalescent plasma for influenza A (H5N1) infection," The New England Journal of Medicine, vol. 357, no. 14, pp. 1450-1451, 2007.

[11] T. C. Luke, E. M. Kilbane, J. L. Jackson, and S. L. Hoffman, "Meta-analysis: convalescent blood products for Spanish influenza pneumonia: a future H5N1 treatment?" Annals of Internal Medicine, vol. 145, no. 8, pp. 599-609, 2006.

[12] Y. Chen, K. Qin, L. W. Wai et al., "Broad cross-protection against $\mathrm{H} 5 \mathrm{~N} 1$ avian influenza virus infection by means of monoclonal antibodies that map to conserved viral epitopes," Journal of Infectious Diseases, vol. 199, no. 1, pp. 49-58, 2009.

[13] B. J. Hanson, A. C. M. Boon, A. P. C. Lim, A. Webb, E. E. Ooi, and R. J. Webby, "Passive immunoprophylaxis and therapy with humanized monoclonal antibody specific for influenza A H5 hemagglutinin in mice," Respiratory Research, vol. 7, article 126, 2006.
[14] N. Prabhu, M. Prabakaran, H.-T. Ho et al., "Monoclonal antibodies against the fusion peptide of hemagglutinin protect mice from lethal influenza A virus $\mathrm{H} 5 \mathrm{~N} 1$ infection," Journal of Virology, vol. 83, no. 6, pp. 2553-2562, 2009.

[15] C. P. Simmons, N. L. Bernasconi, A. L. Suguitan Jr. et al., "Prophylactic and therapeutic efficacy of human monoclonal antibodies against H5N1 influenza," PLoS Medicine, vol. 4, no. 5, article el78, 2007.

[16] R. Wang, A. Song, J. Levin et al., “Therapeutic potential of a fully human monoclonal antibody against influenza A virus M2 protein," Antiviral Research, vol. 80, no. 2, pp. 168-177, 2008.

[17] M. Throsby, E. van den Brink, M. Jongeneelen et al., "Heterosubtypic neutralizing monoclonal antibodies cross-protective against $\mathrm{H} 5 \mathrm{~N} 1$ and $\mathrm{H} 1 \mathrm{~N} 1$ recovered from human IgM+ memory B cells," PLoS ONE, vol. 3, no. 12, Article ID e3942, 2008.

[18] K. Droebner, E. Haasbach, C. Fuchs et al., "Antibodies and CD4+ T-cells mediate cross-protection against $\mathrm{H} 5 \mathrm{~N} 1$ influenza virus infection in mice after vaccination with a low pathogenic H5N2 strain," Vaccine, vol. 26, no. 52, pp. 6965-6974, 2008.

[19] M. R. Sandbulte, G. S. Jimenez, A. C. M. Boon, L. R. Smith, J. J. Treanor, and R. J. Webby, "Cross-reactive neuraminidase antibodies afford partial protection against $\mathrm{H} 5 \mathrm{~N} 1$ in mice and are present in unexposed humans," PLoS Medicine, vol. 4, no. 2, article e59, 2007.

[20] M. Russell, M. Kilian, and M. Lamm, "Biological activities of IgA," Mucosal Immunology, vol. 225, 1999.

[21] B. Corthésy, "Recombinant immunoglobulin A: powerful tools for fundamental and applied research," Trends in Biotechnology, vol. 20, no. 2, pp. 65-71, 2002.

[22] J. K.-C. Ma, B. Y. Hikmat, K. Wycoff et al., "Characterization of a recombinant plant monoclonal secretory antibody and preventive immunotherapy in humans," Nature Medicine, vol. 4, no. 5, pp. 601-606, 1998.

[23] W. R. Brown, R. W. Newcomb, and K. Ishizaka, "Proteolytic degradation of exocrine and serum immunoglobulins," Journal of Clinical Investigation, vol. 49, no. 7, pp. 1374-1380, 1970.

[24] E. Lindh, "Increased resistance of immunoglobulin A dimers to proteolytic degradation after binding of secretory component," Journal of Immunology, vol. 114, no. 1, 1975.

[25] P. Crottet and B. Corthésy, "Secretory component delays the conversion of secretory IgA into antigen- binding competent $\mathrm{F}(\mathrm{ab})$ 2: a possible implication for mucosal defense," Journal of Immunology, vol. 161, no. 10, pp. 5445-5453, 1998.

[26] S. D. Dallas and R. D. Rolfe, "Binding of Clostridium difficile toxin A to human milk secretory component," Journal of Medical Microbiology, vol. 47, no. 10, pp. 879-888, 1998.

[27] A. Phalipon, A. Cardona, J.-P. Kraehenbuhl, L. Edelman, P. J. Sansonetti, and B. Corthésy, "Secretory component: a new role in secretory IgA-mediated immune exclusion in vivo," Immunity, vol. 17, no. 1, pp. 107-115, 2002.

[28] O. Launay, S. Grabar, F. Bloch et al., "Effect of sublingual administration of interferon- $\alpha$ on the immune response to influenza vaccination in institutionalized elderly individuals," Vaccine, vol. 26, no. 32, pp. 4073-4079, 2008.

[29] N. Hagenaars, E. Mastrobattista, H. Glansbeek et al., "Headto-head comparison of four nonadjuvanted inactivated cell culture-derived influenza vaccines: effect of composition, spatial organization and immunization route on the immunogenicity in a murine challenge model," Vaccine, vol. 26, no. 51, pp. 6555-6563, 2008. 
[30] R. Weltzin, S. A. Hsu, E. S. Mittler, K. Georgakopoulos, and T. P. Monath, "Intranasal monoclonal immunoglobulin A against respiratory syncytial virus protects against upper and lower respiratory tract infections in mice," Antimicrobial Agents and Chemotherapy, vol. 38, no. 12, pp. 2785-2791, 1994.

[31] F. M. Apter, W. I. Lencer, R. A. Finkelstein, J. J. Mekalanos, and M. R. Neutra, "Monoclonal immunoglobulin A antibodies directed against cholera toxin prevent the toxin-induced chloride secretory response and block toxin binding to intestinal epithelial cells in vitro," Infection and Immunity, vol. 61, no. 12, pp. 5271-5278, 1993.

[32] G. Liu, X. Chen, J. Xu, C. Yu, H. Song et al., "Construction of dicistronic expression vector in mammalian cell with IRES and dhfr," Bulletin of the Academy of Military Medical Sciences, vol. 24, article 4, 2000.

[33] B. Zhang, X. An, X. Zhang et al., "Cloning of genes by genomic DNA splicing for secretory IgA production," China Biotechnology, vol. 28, pp. 1-6, 2008.

[34] X. An, J. Lu, J.-D. Huang et al., "Rapid assembly of multiple-exon cDNA directly from genomic DNA," PLoS ONE, vol. 2, no. 11, Article ID el179, 2007.

[35] B.-Z. Zhang, X. Zhang, X.-P. An et al., "An easy-to-use sitedirected mutagenesis method with a designed restriction site for convenient and reliable mutant screening," Journal of Zhejiang University B, vol. 10, no. 6, pp. 479-482, 2009.

[36] L. J. Reed and H. Muench, "A simple method of estimating fifty per cent endpoints," American Journal of Epidemiology, vol. 27, no. 3, pp. 493-497, 1938.

[37] K. R. Chintalacharuvu and S. L. Morrison, "Production of secretory immunoglobulin A by a single mammalian cell," Proceedings of the National Academy of Sciences of the United States of America, vol. 94, no. 12, pp. 6364-6368, 1997.

[38] T. H. Weller, "Cytomegaloviruses: a historical perspective," Herpes, vol. 7, no. 3, pp. 66-69, 2000.

[39] J. K.-C. Ma, A. Hiatt, M. Hein et al., "Generation and assembly of secretory antibodies in plants," Science, vol. 268, no. 5211, pp. 716-719, 1995.

[40] F. E. Johansen, I. Natvig Norderhaug, M. Roe, I. Sandlie, and P. Brandtzaeg, "Recombinant expression of polymeric IgA: incorporation of $\mathrm{J}$ chain and secretory component of human origin," European Journal of Immunology, vol. 29, pp. 1701-1708, 1999.

[41] J. Berdoz, C. T. Blanc, M. Reinhardt, J.-P. Kraehenbuhl, and B. Corthésy, "In vitro comparison of the antigen-binding and stability properties of the various molecular forms of IgA antibodies assembled and produced in CHO cells," Proceedings of the National Academy of Sciences of the United States of America, vol. 96, no. 6, pp. 3029-3034, 1999.

[42] T. Uetsuki, A. Naito, S. Nagata, and Y. Kaziro, "Isolation and characterization of the human chromosomal gene for polypeptide chain elongation factor- $1 \alpha$," Journal of Biological Chemistry, vol. 264, no. 10, pp. 5791-5798, 1989.

[43] W. K. Dong, E. H. Chudler, and Y. Kawakami, “Tooth pulpevoked potentials in the trigeminal brain stem nuclear complex," Brain Research, vol. 529, no. 1-2, pp. 131-142, 1990.

[44] R. J. Jackson, M. T. Howell, and A. Kaminski, "The novel mechanism of initiation of picornavirus RNA translation," Trends in Biochemical Sciences, vol. 15, no. 12, pp. 477-483, 1990.

[45] P. Bernstein, S. W. Peltz, and J. Ross, "The poly(A)-poly(A)binding protein complex is a major determinant of mRNA in vitro," Molecular and Cellular Biology, vol. 9, no. 2, pp. 659-670, 1989.
[46] A. Valsamakis, S. Zeichner, S. Carswell, and J. C. Alwine, "The human immunodeficiency virus type 1 polyadenylylation signal: a 3' long terminal repeat element upstream of the AAUAAA necessary for efficient polyadenylylation," Proceedings of the National Academy of Sciences of the United States of America, vol. 88, no. 6, pp. 2108-2112, 1991.

[47] M. Huang and C. Gorman, "The simian virus 40 small-t intron, present in many common expression vectors, leads to aberrant splicing," Molecular and Cellular Biology, vol. 10, no. 4, pp. 18051810, 1990.

[48] M. J. Evans and R. C. Scarpulla, "Introns in the $3^{\prime}$-untranslated region can inhibit chimeric CAT and $\beta$-galactosidase gene expression," Gene, vol. 84, no. 1, pp. 135-142, 1989.

[49] B. Brondyk and D. Groskreutz, "pCI and pSI mammalian expression vectors," Promega Notes 49, 1994.

[50] R. J. Kaufman, L. C. Wasley, A. J. Spiliotes et al., "Coamplification and coexpression of human tissue-type plasminogen activator and murine dihydrofolate reductase sequences in Chinese hamster ovary cells," Molecular and Cellular Biology, vol. 5, no. 7, pp. 1750-1759, 1985.

[51] R. J. Kaufman and P. A. Sharp, "Growth-dependent expression of dihydrofolate reductase mRNA from modular cDNA genes," Molecular and Cellular Biology, vol. 3, no. 9, pp. 1598-1608, 1983. 




The Scientific World Journal
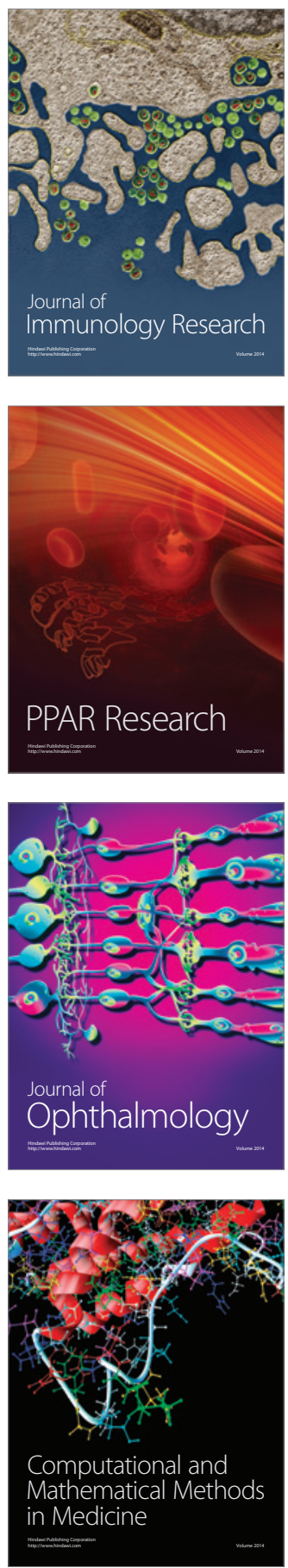

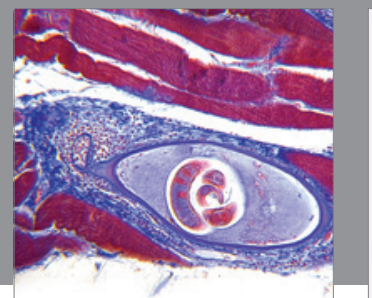

Gastroenterology

Research and Practice
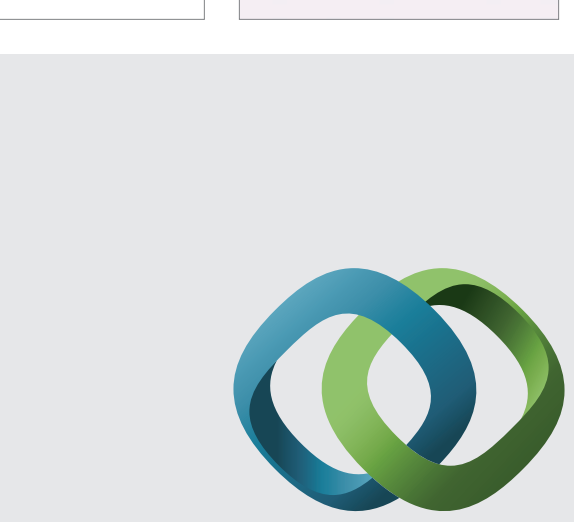

\section{Hindawi}

Submit your manuscripts at

http://www.hindawi.com
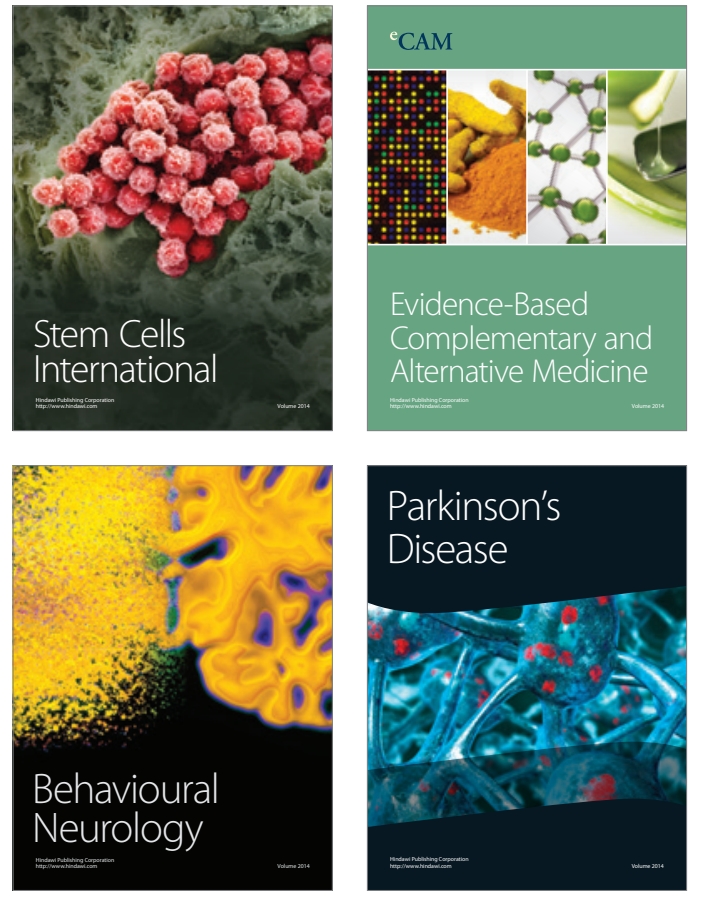
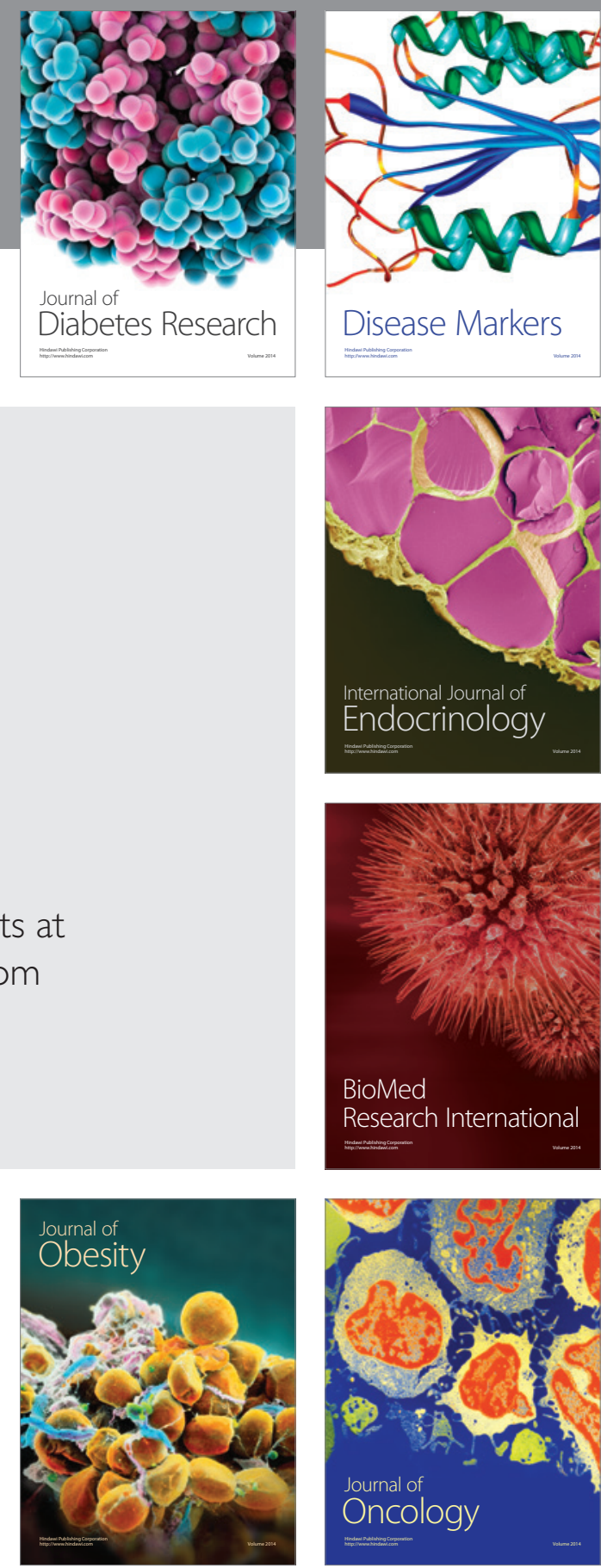

Disease Markers
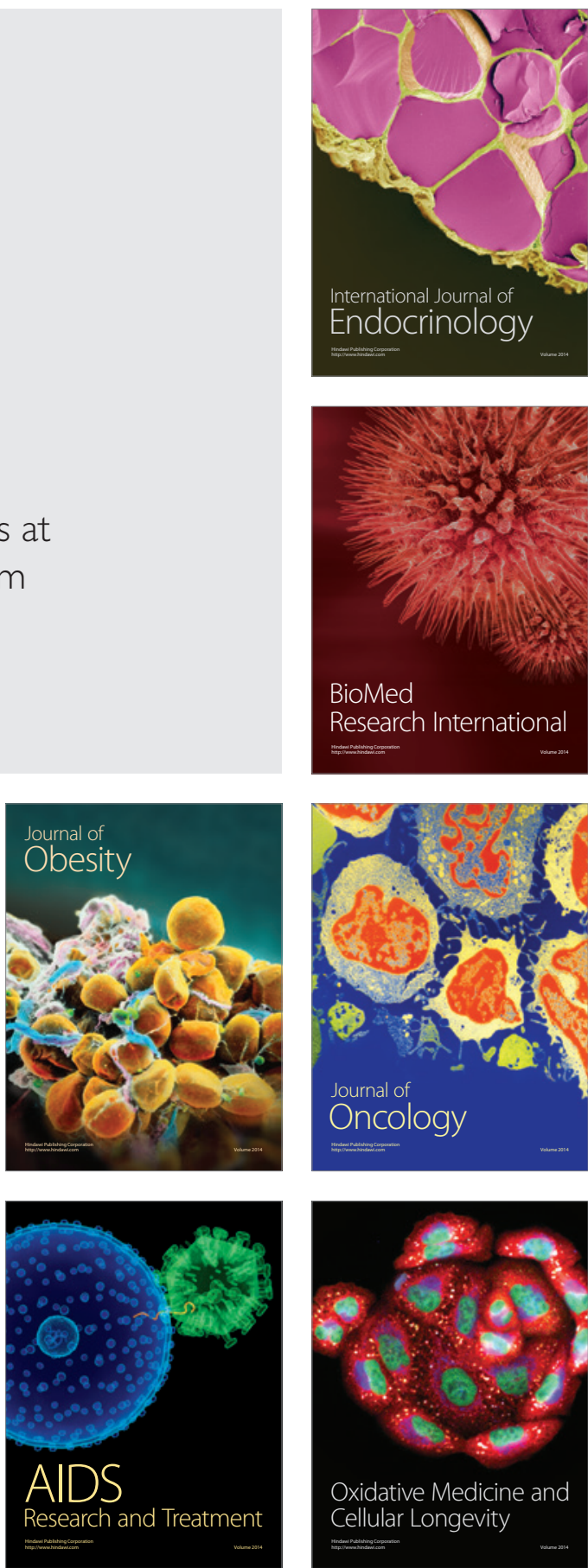